\title{
Prolactin and silicone breast implants: It takes two to tango
}

\author{
Borba VV $V^{1,2,3}$ and Shoenfeld $Y^{3,4,5 *}$ \\ ${ }^{1}$ Department 'A' of Internal Medicine, Coimbra University Hospital Centre, Coimbra, Portugal \\ ${ }^{2}$ Faculty of Medicine, University of Coimbra, Coimbra, Portugal \\ ${ }^{3}$ Zabludowicz Center for Autoimmune Diseases, Sheba Medical Center, Tel-Hashomer, Israel \\ ${ }^{4}$ Sackler Faculty of Medicine, Tel-Aviv University, Tel-Aviv, Israel \\ ${ }^{5}$ Head of "The Mosaic of Autoimmunity” Project in Saint Petersburg University, Russia
}

Autoimmune diseases affect approximately $5-20 \%$ of the developed world population and represent a significant cause of morbidity and mortality among young adults. The etiopathogenesis of autoimmune diseases comprises a combination of genetic, immune, hormonal, and environmental factors, so-called mosaic of autoimmunity. Recently, Shoenfeld et al. recognized that different conditions linked to exposure to adjuvants resulted in similar complexes of signs and symptoms and proposed to label this condition "ASIA" (autoimmune/inflammatory syndrome induced by adjuvants) [1], which illustrates the role of environmental immune stimulating agents, or adjuvants, in the instigation of complex autoimmune reactions among individuals bearing a genetic preponderance for autoimmunity [2]. Nowadays, more than 300 patients are listed in the ASIA international registry $[3,4]$. Although the link between silicon exposure and the development of autoimmune phenomena has been postulated long time ago, there is a growing evidence in the recent literature, supporting this association essentially based on case reports of patients carrying silicone prosthesis [5]. Breast implants contain a type of medical silicone called polydimethylsiloxane (PDMS), which has been widely used in the manufacture of assorted medical devices and, have long been considered biologically inert and harmless. Since the introduction of silicone breast implants in the early 1960s, more than 10 million patients worldwide underwent this procedure, especially in the field of reconstructive surgery. Several cohort studies declare the development of non-specific symptoms like myalgia, chronic fatigue, arthralgia, among others and/or the flowering of autoimmune diseases such as systemic sclerosis, systemic lupus erythematosus, rheumatoid arthritis, and Sjögren's syndrome after the implantation on silicone breast prosthesis [58]. A combination of a genetic predisposition (HLA-DR4, DRB1, DR53, DQA $1^{\star} 0102$, HLA-C), relevant environmental triggers, history of allergic conditions, established autoimmune diseases or prior documented autoimmune reaction to an adjuvant (implants, vaccines) were considered risk factors for the occurrence of a hyperactive immune response. Obviously, the assumption that an autoimmune disorder would prosper with no relation with the silicon is plausible. Nevertheless, studies found that after replacing siliconefilled by saline-cellulose-mixed implants, approximately $75 \%$ of the patients experienced a strong improvement of their complaints [7]. In light of the adjuvant effect of silicones, a few pathways may justify this connection. Silicone leakage, also defined as 'silicone bleeding', represents the migration of relatively low molecular weight silicone compounds through the implant elastomer envelope and may cause a histiocytic reaction with foreign body giant cells forming granulomas. It could increase the exposure of self-antigens by providing a hydrophobic surface to change the conformation of those proteins thus causing recognition by the immune system and subsequent autoimmune reaction. In addition, it may induce an immunogenic response via cross-reactivity with glucosaminoglycans, a silicon-containing molecule found in connective tissue [9]. On the other hand, several case reports describe postoperative galactorrhea after aesthetic breast augmentation. The skin incision, the breast manipulation and particularly, the compression of the mammary gland by the implant results in stimulating signal to the hypophysis, and therefore increasing prolactin secretion [10,11]. Years after the procedure, persistent hyperprolactinemia has been documented among those patients, without any other identified cause besides the breast implantation surgery [12]. Prolactin has a recognized immunestimulatory effect, promoting autoimmune phenomena. The prolactin receptor, which is a member of the type 1 cytokine/hematopoietic receptor super-family and is widely expressed through the immune system. Binding of prolactin to its receptor activates downstream signaling pathways that will manipulate immune cells proliferation, differentiation, secretion and survival. In addition, elevated serum levels of prolactin impair B cell clonal deletion, deregulate receptor editing and decrease the threshold for activation of anergic B cells, promoting aberrant reactivity [13]. High prolactin levels have been related in several autoimmune diseases, frequently influencing disease development and perpetuation [14]. In conclusion, silicone breast implants could represent a strong arouse for the development of autoimmune disorders, not only by the surgical procedure per se, and the mechanical compression of the implant, stimulating prolactin secretion, but also silicone molecules acting as an adjuvant to abnormal immune reactions. Accordingly, it stands to reason that women with recognized predisposition for autoimmune diseases should be advised by the physicians to avoid silicone breast implantation $[7,15]$.

\section{Conflicts of interest}

The authors have no potential conflicts of interest in authorship or publication.

${ }^{\star}$ Correspondence to: Yehuda Shoenfeld, Zabludowicz Center for Autoimmune Diseases, Sheba Medical Center (Affiliated to Tel-Aviv University), Tel: 265601, Israel, E-mail: shoenfel@post.tau.ac.il

Key words: autoimmune disease, autoimmune/inflammatory syndrome induced by adjuvants, breast implant, adjuvant, silicone, prolactin, galactorrhea, bromocriptine

Received: February 18, 2019; Accepted: March 08, 2019; Published: March 15, 2019 


\section{Financial declaration}

The authors have no relevant financial disclosures.

\section{References}

1. Cohen Tervaert JW (2018) Autoinflammatory/autoimmunity syndrome induced by adjuvants (ASIA; Shoenfeld's syndrome): A new flame. Autoimmun Rev 17: 12591264. [Crossref]

2. Segal Y, Dahan S, Sharif K, Bragazzi NL, Watad A, et al. (2018) The value of autoimmune syndrome induced by adjuvant (asia) - shedding light on orphan diseases in autoimmunity. Autoimmunity reviews 17: 440-448 [Crossref]

3. Goren I, Segal G, Shoenfeld Y (2015) Autoimmune/inflammatory syndrome induced by adjuvant (asia) evolution after silicone implants. Who is at risk? Clinical rheumatology 34: 1661-1666. [Crossref]

4. Watad A, Quaresma M, Bragazzi NL, Cervera R, Tervaert JWC, et al. (2018) The autoimmune/inflammatory syndrome induced by adjuvants (asia)/shoenfeld's syndrome: Descriptive analysis of 300 patients from the international asia syndrome registry. Clinical rheumatology 37: 483-493. [Crossref]

5. Watad A, Rosenberg V, Tiosano S (2018) Silicone breast implants and the risk of autoimmune/rheumatic disorders: a real-world analysis. Int J Epidemiol 47: 18461854. [Crossref]

6. Nunes ESD, Grundler C, Spengler M, Horimoto AMC, Machado MA, et al. (2017) Autoimmune syndrome induced by adjuvants (asia) after silicone breast augmentation surgery. Plast Reconstr Surg Glob Open 5: e1487 [Crossref]
7. Cohen Tervaert JW, Colaris MJ, van der Hulst RR (2017) Silicone breast implants and autoimmune rheumatic diseases: myth or reality. Curr Opin Rheumatol 29: 348-354. [Crossref]

8. Pavlov-Dolijanovic S, Vujasinovic Stupar N (2017) Women with silicone breast implants and autoimmune inflammatory syndrome induced by adjuvants: Description of three patients and a critical review of the literature. Rheumatology international 37 : 1405-1411. [Crossref]

9. Colaris MJL, de Boer M, van der Hulst RR, Cohen Tervaert JW (2017) Two hundreds cases of Asia syndrome following silicone implants: A comparative study of 30 years and a review of current literature. Immunologic research 65: 120-128. [Crossref]

10. Ayestaray B, Dudrap E, Chaibi A (2011) Galactorrhea after aesthetic breast augmentation with silicone implants: Report of two cases and management of postoperative galactorrhea. Aesthetic plastic surgery 35: 408-413. [Crossref]

11. Yang EJ, Lee KT, Pyon JK, Bang SI (2012) Treatment algorithm of galactorrhea after augmentation mammoplasty. Annals of plastic surgery 69: 247-249. [Crossref]

12. Marino A, Patel T, Whitten E, Peiris AN (2009) Persistent hyperprolactinemia following mammoplasty: A rare association. Tennessee medicine 102: 47-48. [Crossref]

13. Peeva E, Zouali M (2005) Spotlight on the role of hormonal factors in the emergence of autoreactive b-lymphocytes. Immunology letters 101: 123-143. [Crossref]

14. Borba VV, Zandman-Goddard G, Shoenfeld Y (2018) Prolactin and Autoimmunity. Front Immunol 9: 73. [Crossref]

15. de Boer M, Colaris M, van der Hulst RRWJ (2017) Is explanation of silicone breast implants useful in patients with complaints? Immunol Res 65: 25-36. [Crossref]

Copyright: $\odot 2019$ Borba VV. This is an open-access article distributed under the terms of the Creative Commons Attribution License, which permits unrestricted use, distribution, and reproduction in any medium, provided the original author and source are credited. 